\title{
Multifunctional biodegradable polymer/clay nanocomposites with antibacterial properties in drug delivery systems
}

\author{
ALICJA RAPACZ-KMITA ${ }^{1 *}$, BARBARA SZARANIEC ${ }^{1}$, MACIEJ MIKOŁAJCZYK ${ }^{2}$, \\ EWA STODOLAK-ZYCH ${ }^{1}$, EWA DZIERZKOWSKA ${ }^{1}$, MARCIN GAJEK $^{1}$, PIOTR DUDEK ${ }^{3}$ \\ ${ }^{1}$ AGH University of Science and Technology, Faculty of Materials Science and Ceramics, Kraków, Poland. \\ ${ }^{2}$ The University Hospital in Krakow, Division of Microbiology, Kraków, Poland. \\ ${ }^{3}$ AGH University of Science and Technology, Faculty of Mechanical Engineering and Robotics, Kraków, Poland.
}

\begin{abstract}
Purpose: The aim of this study was to investigate the possibility of intercalation of gentamicin and neomycin in montmorillonite (MMT) nanofillers, as well as to study the in vitro antimicrobial properties of nanocomposite films containing a small amount of thus obtained nanofillers. Methods: The polylactide matrix (PLA) nanocomposite films with drug-intercalated montmorillonite fillers were obtained by casting after intercalation of drugs in aqueous solutions. The efficiency of intercalation has been confirmed by X-ray diffraction (XRD) and Zeta potential measurements. The materials were studied for surface wettability, roughness and mechanical properties during 6 weeks of incubation in phosphate buffer saline, and their bactericidal activity was tested against Escherichia coli bacteria before and after 6 weeks of incubation in distilled water at $37^{\circ} \mathrm{C}$. The presence of antibiotics during the incubation was monitored by conductivity and $\mathrm{pH}$ measurements. Results: The results indicate that nanocomposite polylactide films with montmorillonite filler intercalated with gentamicin and neomycin tend to degrade faster that their counterparts with non-intercalated fillers, which affects their mechanical properties. However, drug intercalation provided an antibacterial activity, which was confirmed by the presence of zones inhibiting the growth of Gram-negative bacteria for both antibiotics. It was also confirmed that the interaction of antibiotics with clay and polymer matrix did not adversely affect this bactericidal effect. Conclusions: Montmorillonite can be successfully intercalated with both gentamicin and neomycin, and then used as active filler for polylactide films having very good antibacterial properties, therefore their use in biomedical applications can be significantly expanded.
\end{abstract}

Key words: nanocomposite, clay mineral, drug carrier, mechanical properties, antimicrobial activities

\section{Introduction}

Montmorillonite (MMT) is a frequently used nano-additive in polymer matrix nanocomposites due to the possibility of modifying its structure, such as complete exfoliation or partial expansion of the space between octahedral layers, which creates conditions for the intercalation of numerous active substances. In the case of exfoliation MMT, even a small addition to the polymer matrix can positively affect the mechanical properties, increase thermal resistance, and, when using biodegradable polymers, improve the biodegradability of the composite [21], [19]. In turn, inter- calated MMT, due to the fact that it is non-toxic, chemically inert and stable in both acidic and alkaline environments, is often used in drug delivery systems, most often acts as a carrier that can be used alone or in combination with polymers [14]. High interest in the use of montmorillonite for this purpose has resulted in numerous literature data on its use in the distribution of antibiotics [18], [11]. It is known, among others, that antibiotics such as gentamicin $(\mathrm{G})$ or metronidazole (MTZ) do not lose their antibacterial properties in combination with montmorillonite, and, for example, the introduction of gentamicin between the MMT layers results in longer antibiotic release [18]. It was also found that the MMT-MTZ complex provides

\footnotetext{
* Corresponding author: Alicja Rapacz-Kmita, AGH University of Science and Technology, Faculty of Materials Science and Ceramics, ul. Mickiewicza 30, 30-059 Kraków, Poland. Phone: +48 12 6172329, fax: +48 12 6331593, e-mail address: kmita@agh.edu.pl

Received: March 2nd, 2020

Accepted for publication: April 6th, 2020
} 
extended release of the drug in synthetically produced intestinal fluid, better retention and bioavailability compared to pure MTZ [11]. In addition to antibiotics, anti-depressant drugs [16], anti-diabetic [6], enzymes and proteins (pepsin, hemoglobin, lactoglobulin) [13] as well as vitamins were also introduced into the MMT structure. Experiments were also carried out to intercalate vitamin B1 to synthetic montmorillonite structures in controlled drug delivery systems [8].

A separate aspect is related to the issue of obtaining the antibacterial properties of polymeric materials resulting from the combination of polymer and MMT, the effectiveness of which is achieved by modifying the polymer matrix (biopolymers or biodegradable polymers) using MMT-pharmaceutical conjugates. Each configuration has other unique properties, and an example of such material is MMT-polymer nanocomposites, which can be used as a mucoadhesive drug carrier [24], [9] or act as both active substance delivery agents, have antibacterial properties, and modifiers that increase nanocomposite strength [20].

Controlled release of the drug as well as the possibility of treating the side effects associated with taking the pharmaceutical was also noted in the case of montmorillonite combined with biodegradable polymers, which was intercalated with anticancer drugs such as Docetaxel administered orally [7]. A comparable effect as well as improved drug distribution was also achieved in the case of MMT intercalation 5-fluorouracil in a polymer matrix [1]. Similarly, the with presence of montmorillonite in the MMT-dexamethason conjugate (a drug with long-lasting and potent antiinflammatory action) resulted in a longer drug release time and increased strength of the nanocomposite with the ethylene and vinyl acetate copolymer [3], [5].

The positive effect of MMT as a drug carrier in the polymer matrix is also observed for the group of non-steroidal anti-inflammatory drugs. The combination of montmorillonite, guar gum and ibuprofen initially showed a reduction in the release effect of the drug and then a sustained release in intestinal fluid at $\mathrm{pH} 7.4$ [5]. In turn, sodium naproxen (NAP) in polyurethane nanocomposites in hydrogels, containing organically modified montmorillonite that slows naproxen release, has been recognized as a modern wound dressing that can cause a significant reduction in edema [15]. It was also discovered that a polyoxypropylene-montmorillonite nanocomposite containing ibuprofen and theophylline can be used as a carrier for prolonged release of drugs in the treatment of colon diseases [22].

The aim of this study was to investigate the effectiveness of drug intercalation in the montmorillonite nanofiller and its influence on the resulting antimicrobial properties in vitro of nanocomposite films filled with a relatively small amount of the intercalated montmorillonite-based conjugates. Two antibiotics, showing a bactericidal effect in relation to Gramnegative bacteria, namely: gentamicin $(\mathrm{G})$ and neomycin $(\mathrm{N})$, were used as the intercalates, and finally the structural, physicochemical and mechanical properties of the films, as well as their degradability in vitro were also examined. Ultimately, the utilitarian purpose of this study was to check the applicability of biodegradable antibacterial films in medical applications, such as membranes and textiles for wound healing, cell scaffolding as well as small devices and temporary implants.

\section{Materials and methods}

\subsection{Materials}

Biodegradable poly-l-lactide (PLA) INGEO $3251 \mathrm{D}$ (Nature-Works USA) was used in this study as a matrix of nanocomposites filled with hybrid MMT conjugates intercalated with two aminoglycoside antibiotics, to obtain antibacterial properties of the PLA/MMT, $\mathrm{PLA} / \mathrm{MMT}+\mathrm{G}$ and PLA/MMT+N nanocomposite materials in the form of films. Montmorillonite (MMT) Veegum ${ }^{\circledR}$ F (R.T. Vanderbilt Company, Inc., USA) was used as a drug carrier for antibiotics, showing a bactericidal effect especially in relation to Gram-negative bacteria, namely: gentamicin $(\mathrm{G})$ in the form of gentamicin sulfate with the chemical formula $\mathrm{C}_{21} \mathrm{H}_{43} \mathrm{~N}_{5} \mathrm{O}_{7} \cdot \mathrm{H}_{2} \mathrm{SO}_{4}$ (Pharma-Cosmetic Poland) and neomycin (N) also in the form of a sulphate with the chemical formula $\mathrm{C}_{23} \mathrm{H}_{46} \mathrm{~N}_{6} \mathrm{O}_{13} \cdot 3 \mathrm{H}_{2} \mathrm{SO}_{4}$ (also from Pharma-Cosmetic Poland). The sulphate forms of antibiotics used in the work were chosen deliberately to guarantee their hydrophilicity and ease of dissolution in water, which enabled us to carry out the intercalation process in the water suspension. Dichloromethane (Sigma-Aldrich Poland) and 70\% 2-propanol (Avantor Poland) were also used as additional chemicals.

\subsection{Preparation of MMT + G and MMT $+\mathbf{N}$ hybrid conjugates}

The process of montmorillonite intercalation with both gentamicin and neomycin was carried out in aqueous suspensions, using a magnetic stirrer, at a tem- 
perature of $60{ }^{\circ} \mathrm{C}$ and a mixing time of $24 \mathrm{~h}$. Thus obtained suspensions were dried at $60^{\circ} \mathrm{C}$, and then the powdered conjugates obtained were ground in an agate mortar.

\subsection{Preparation of PLA/MMT, PLA/MMT + G and PLA/MMT + N films}

All the films, i.e., the PLA/MMT reference sample, as well as the PLA/MMT $+\mathrm{G}$ and PLA/MMT + N nanocomposite films were prepared from the polymer solution by solvent casting. In the first step, the lactic acid polymer (PLA) in the form of pellets was dissolved in dichloromethane (DCM, Sigma-Aldrich) in a ratio of 1:10 by weight, and the solution was stirred using a magnetic stirrer (ES manual type Wigo 24) for 6 hours to ensure good dissolution and homogenization. The individual nanofiller slurries were then added to $20 \mathrm{ml}$ of polymer solution ( $2 \mathrm{~g}$ of PLA sample) and mixed. The suspensions after the homogenization step were poured into separate Petri dishes, which were left for 24 hours in a laboratory hood at room temperature to evaporate the solvent, however, to ensure complete evaporation, the samples were then placed in a SPT-200 vacuum furnace. In the nanocomposite materials obtained in the form of a foil, the amount of nanoadditives was about $2 \%$ by weight.

\subsection{Simulation of the degradation process in vitro}

The degradation of all nanocomposite films, PLA/MMT, PLA/MMT + G and PLA/MMT + N, was tested in the 6 weeks in vitro tests at $37{ }^{\circ} \mathrm{C}$ in two types of liquid medium, namely: PBS (phosphate buffered saline) and distilled water. For testing in PBS, the foils were cut into strips with a length of approx. $80 \mathrm{~mm}$ and a width of approx. $5 \mathrm{~mm}$, which were then used after the degradation period to measure the mechanical tensile strength. The squareshaped samples having dimensions of $6 \times 6 \mathrm{~mm}$ as well as circular samples of $6 \mathrm{~mm}$ in diameter were incubated in distilled water, and then subjected to antibacterial tests after an appropriate incubation period. During the incubation of nanocomposites in distilled water, measurements of $\mathrm{pH}$ changes and electrical conductivity were also carried out in order to check the effect of the material on the surrounding environment, the rate of release of drugs into the environment and the degradation of the nanocomposite material.

\subsection{Methods}

\subsubsection{Investigation of the drug intercalated MMT conjugate powders}

\section{$X R D$ analysis}

In order to confirm the montmorillonite intercalation process with gentamicin and neomycin, X-ray diffraction (XRD) measurements of conjugates in the form of powders were conducted. The XRD analysis was carried out in the $3-70^{\circ} 2 \theta$ range using $\mathrm{Cu}-\mathrm{K} \alpha$ radiation and the PANalytical Empyrean diffractometer with a 0.008 degree step (total time about $4 \mathrm{~h}$ ).

\section{Zeta potential}

The Zeta potential measurements were also made on the prepared MMT, MMT/G and MMT/N conjugates. It was used to determine the size of the effective electric charge of powder particles. The obtained conjugates were combined with $100 \mathrm{ml}$ of distilled water and placed in an ultrasonic Techpan UD-20 disintegrator. Thus prepared suspensions were placed in measuring cells and subjected to three measurements of Zeta potential using the Zetasizer Nano SZ Malvern Instruments. The average refractive index for montmorillonite of $1.52 \mathrm{~nm}$ was assumed.

\subsubsection{Investigation of nanocomposite materials}

\section{Surface wettability}

Measurements of wettability of the surface of nanocomposites were made by determining the contact angle to assess the hydrophobicity/hydrophilicity of the obtained materials. This was determined by the drop method in the DSA 10-MK2 drop shape analyzer (Krüss). The droplet was placed on the surface of the nanocomposite and its shape was recorded using a digital camera, and finally analyzed using a spherical fit. The measurements were repeated eight times for the upper (free side) and lower side (mould side) of nanocomposite film samples. The tests were made for both starting materials (before incubation), but also for materials after 6 weeks of in vitro incubation in distilled water. 


\section{Surface roughness}

Surface roughness denotes optically or mechanically detectable surface irregularities that have been determined to evaluate the adhesion between the surface of the nanocomposite and the modifying agents. The material was free from contamination by immersion in ethyl alcohol, placed in the Hommelwerke T1000 profilometer and analyzed under the pressure of a diamond head moving on the surface of the nanocomposite at a constant speed. Eight roughness measurements were made for both the top and bottom sides (mould side) of nanocomposite foils. The measurements were carried out on films before the incubation process in order to evaluate the effect of surface roughness on their antibacterial activity against $E$. coli.

\section{Mechanical properties}

Mechanical properties were determined on the basis of tests carried out using a Zwick-Roller testing machine, type HTO- 02 on strips approximately $8 \mathrm{~cm}$ long and $0.5 \mathrm{~cm}$ wide cut from the obtained PLA/MMT, PLA/MMT $+\mathrm{G}$ and PLA/MMT $+\mathrm{N}$ films. An additional set of samples was also incubated in PBS, and mechanical tests were carried out after 1,2, 3, 4 and 6 weeks of incubation. During testing, tensile strength, elastic modulus and maximum strain at break were determined (test speed of $2 \mathrm{~mm} / \mathrm{min}$ ), in accordance with ISO 527-3, on 15 samples of each material.

\section{Drug release and durability}

Monitoring of $\mathrm{pH}$ changes and ionic conductivity was performed to check the durability of materials and determine the drug release profile during incubation of nanocomposite films for 6 weeks in distilled water at $37^{\circ} \mathrm{C}$. Monitoring of the release of all active substances was carried out by measuring the electrical conductivity and $\mathrm{pH}$ changes of the medium using a conductometer $\mathrm{CC}-411$ and $\mathrm{pH}$-meter CP-401 (ELMETRON). Measurements were performed after reaching room temperature.

\section{Antibacterial properties}

The antibacterial activity of PLA/MMT, PLA/MMT $+\mathrm{G}$ and PLA/MMT $+\mathrm{N}$ films was tested against Gram-negative Escherichia coli (E. coli) bacteria (ATCC 25922) using the Kirby-Bauer disk-diffusion antibiotic susceptibility test. The bacteria were grown in Oxoid's Columbia Blood Agar on Petri dishes at $36 \pm 2{ }^{\circ} \mathrm{C}$ for 24 hours. Three to five colonies were resuspended in physiological $\mathrm{NaCl}$ solution $(0.9 \%)$ to obtain a suspension of $0.5 \mathrm{McF}$ arland (approximately $1.5 \cdot 10^{8} \mathrm{CFU}$ ). The prepared suspension was inoculated with a sterile swab on a medium (Mueller Hinton Agar Oxoid) in vessels with a diameter of $100 \mathrm{~mm}$. Prior to antibacterial testing, disc samples with a diameter of $6 \mathrm{~mm}$ cut from foil were sterilized with alcohol and dried in a laboratory drier at a temperature of about $50{ }^{\circ} \mathrm{C}$ for 24 hours to evaporate the alcohol, and then placed on the inoculated substrate. After 24 hours of incubation at $36 \pm 2{ }^{\circ} \mathrm{C}$ under aerobic conditions, the diameters of the bacterial growth inhibition zones were measured using a caliper for each type of nanocomposite film, on 6 discs, and the average of 6 measurements was considered a result. Measurements of antibacterial activity of the films were performed in the initial state (before incubation) as well as after a 6-week incubation in distilled water to confirm bactericidal properties and the presence of antibiotics as well as its biocidal properties after incubation. Samples were placed both smooth and rough sides in the direction of agar with bacterial culture, so as to obtain information on the effect of the nature of the surface on the amount of antibiotic efficiently released from the film.

\section{Results}

\subsection{Investigation of drug-intercalated MMT powder conjugates}

In Table 1 , the comparison of $d_{001}$ values for raw material (MMT) and reference sample (MMT $+\mathrm{H}_{2} \mathrm{O}$ ) as well as for MMT $+\mathrm{G}$ and MMT $+\mathrm{N}$ conjugates are presented. The MMT $+\mathrm{H}_{2} \mathrm{O}$ reference sample was tested to determine the effect of the presence of water in the MMT interlayer on the $d_{001}$ distance.

Table 1. Results of measurements of $d_{001}$ values for powder materials

\begin{tabular}{|l|c|}
\hline \multicolumn{1}{|c|}{ Powder } & $d_{001}[\AA]$ \\
\hline MMT & 11.289 \\
\hline $\mathrm{MMT}+\mathrm{H}_{2} \mathrm{O}$ & 11.842 \\
\hline $\mathrm{MMT}+\mathrm{G}$ & 15.462 \\
\hline $\mathrm{MMT}+\mathrm{N}$ & 16.476 \\
\hline
\end{tabular}

Zeta potential values were measured for the MMT $+\mathrm{H}_{2} \mathrm{O}$ reference powder, as well as for MMT $+\mathrm{G}$ and MMT $+\mathrm{N}$ at $25^{\circ} \mathrm{C}$, and the results are summarized in Table 2. Intercalation of montmorillonite with drugs changes its electrical potential from negative to positive, which also indicates on the effectiveness of the intercalation process. 
Table 2. Results of Zeta and $\mathrm{pH}$ potential measurements for powders

\begin{tabular}{|l|c|c|}
\hline \multicolumn{1}{|c|}{ Powder } & Zeta potential $[\mathrm{mV}]$ & $\mathrm{pH}$ \\
\hline $\mathrm{MMT}$ & $-34.4 \pm 0.5$ & 8.1 \\
\hline $\mathrm{MMT}+\mathrm{H}_{2} \mathrm{O}$ & $-35.8 \pm 0.7$ & 8.6 \\
\hline $\mathrm{MMT}+\mathrm{G}$ & $12.3 \pm 0.3$ & 5.7 \\
\hline $\mathrm{MMT}+\mathrm{N}$ & $11.7 \pm 0.3$ & 5.8 \\
\hline
\end{tabular}

\subsection{Investigation of nanocomposite foil materials}

\section{Surface wettability}

The mean values of the contact angle measured for nanocomposite films before and after a 6 weeks incubation in distilled water are shown in Table 3 . The contact angle before incubation was measured on both sides of the obtained films, and the upper and lower sides of the nanocomposite denote the "free surface" which does not contact the vessel and the opposite surface in contact with the vessel in which the foils were cast. After a 6-week incubation, however, the upper and lower surfaces could not be recognized as a result of degradation, and thus only one average was calculated based on the surface wettability measurements.

Table 3. Results of measurements of the contact angle of nanocomposite foils

\begin{tabular}{|c|l|c|c|}
\hline \multirow{2}{*}{ Foil } & \multicolumn{1}{|c|}{$\begin{array}{c}\text { Side/ } \\
\text { Surface }\end{array}$} & $\begin{array}{c}\text { Contact } \\
\text { angle } \theta\left[^{\circ}\right]\end{array}$ & $\begin{array}{c}\text { Contact angle after } \\
6 \text { weeks of incubation } \\
\text { in distilled water } \theta\left[^{\circ}\right]\end{array}$ \\
\hline \multirow{2}{*}{ PLA/MMT } & Upper & $81.45 \pm 3.20$ & \multirow{2}{*}{$67.33 \pm 6.15$} \\
\cline { 2 - 3 } & Lower & $75.10 \pm 3.12$ & \\
\hline PLA/MMT + G & Upper & $80.96 \pm 5.18$ & \multirow{2}{*}{$54.01 \pm 4.43$} \\
\cline { 2 - 3 } & Lower & $68.40 \pm 2.97$ & \\
\hline PLA/MMT + N & Upper & $83.65 \pm 5.33$ & \multirow{2}{*}{$64.94 \pm 3.01$} \\
\cline { 2 - 3 } & Lower & $77.40 \pm 5.32$ & \\
\hline
\end{tabular}

\section{Surface roughness}

The surface roughness was measured as a parameter that may be of importance in contact with the bacterial culture due to the complexity of the contact surface and the resulting antibacterial response. The following parameters of the nanocomposite surface roughness were examined: $R_{a}$ (arithmetic mean of profile deviation), $R_{t}$ (maximum height between the highest and lowest point on the sample) and $R_{z}$ (height of the roughness profile by the highest 5 and the lowest 5 points on the sample). The obtained mean values and the resultant standard deviation are presented in Table 4.
Table 4. Roughness of nanocomposite foils

\begin{tabular}{|l|l|c|c|c|}
\hline \multicolumn{1}{|c|}{ Foil } & $\begin{array}{c}\text { Side/ } \\
\text { Surface }\end{array}$ & $R_{a}[\mu \mathrm{m}]$ & $R_{t}[\mu \mathrm{m}]$ & $R_{z}[\mu \mathrm{m}]$ \\
\hline \multirow{2}{*}{ PLA/MMT } & Upper & $0.96 \pm 0.16$ & $7.83 \pm 2.14$ & $4.91 \pm 0.59$ \\
\cline { 2 - 5 } & Lower & $0.89 \pm 0.13$ & $9.25 \pm 1.53$ & $0.96 \pm 0.16$ \\
\hline $\begin{array}{l}\text { PLA/MMT } \\
+\mathrm{G}\end{array}$ & Upper & $3.23 \pm 0.48$ & $33.11 \pm 8.72$ & $17.94 \pm 2.60$ \\
\cline { 2 - 5 } $\begin{array}{l}\text { PLA/MMT } \\
\text { + N }\end{array}$ & Upper & $1.53 \pm 0.34$ & $19.55 \pm 6.64$ & $10.11 \pm 2.08$ \\
\cline { 2 - 5 } & Lower & $0.79 \pm 0.24$ & $18.48 \pm 4.62$ & $12.02 \pm 2.74$ \\
\hline
\end{tabular}

\section{Mechanical properties}

Mechanical performance of nanocomposite foils was monitored throughout the entire degradation process, and the summarized results are shown in Figs. 1-3. It is clearly visible that the most pronounced changes occurred in the initial stage of degradation test and are the most distinct in the PLA/MMT+N material, which correlates with the highest interlayer space of montmorillonite in that material. It may be explained by the fact that although partial exfoliation of the MMT clay occurred, which should lead to a better ability to reinforce the polymer matrix, intercalation took place after this process, and the interlayer space is partially occupied by drug molecules, so the polymer chains cannot penetrate and interlock there. Resulting interfaces are week, which leads to a faster deterioration of mechanical properties during the degradation, which is noticeable during first four weeks of the test.

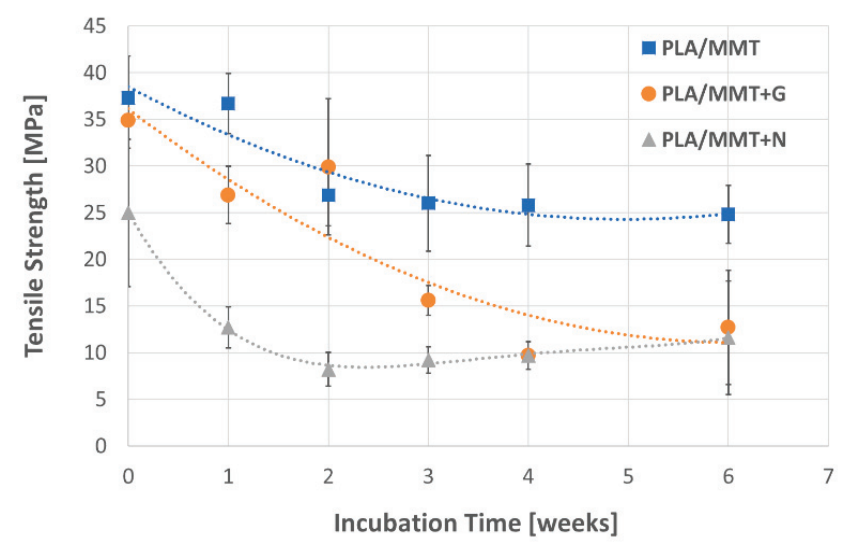

Fig. 1. Changes in tensile strength of nanocomposite films during the degradation test

After this period, dynamics of mentioned deterioration decreases and stabilizes at the level similar to the plain PLA/MMT nanocomposite foil without the drug intercalated. Similar trend can be observed for changes in maximum strain of the foils during the degradation test and they are again correlated the most probably with height of interlaminar distance and available space. It is also visible that, in general, materials become less deformable and more brittle so the 
resulting strain is lower. It is also well reflected in the results of the tensile modulus of elasticity, but the increasing brittleness and lower strain eventually causes even increase in the stiffness of the materials tested, so degraded foils even exhibit relative increase in the values of the elastic modulus at the end of the 6-weeks degradation test.

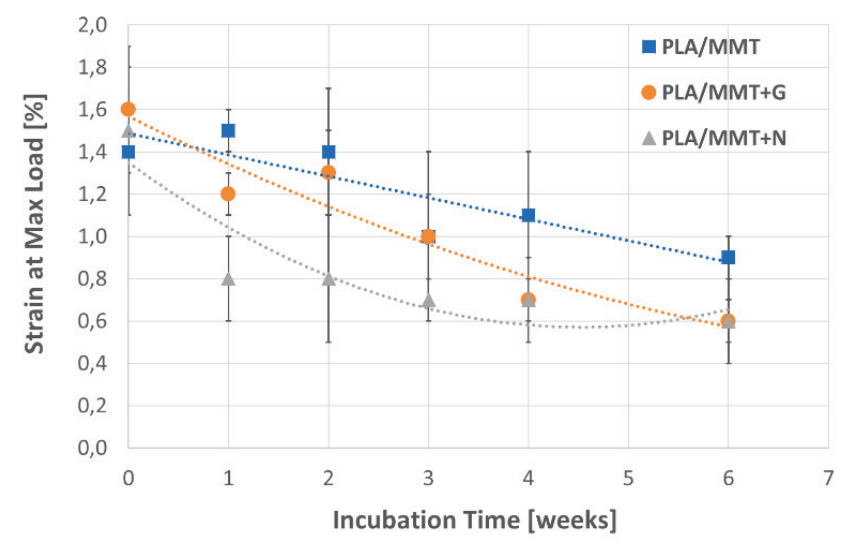

Fig. 2. Changes in strain at max load of nanocomposite foils during degradation test

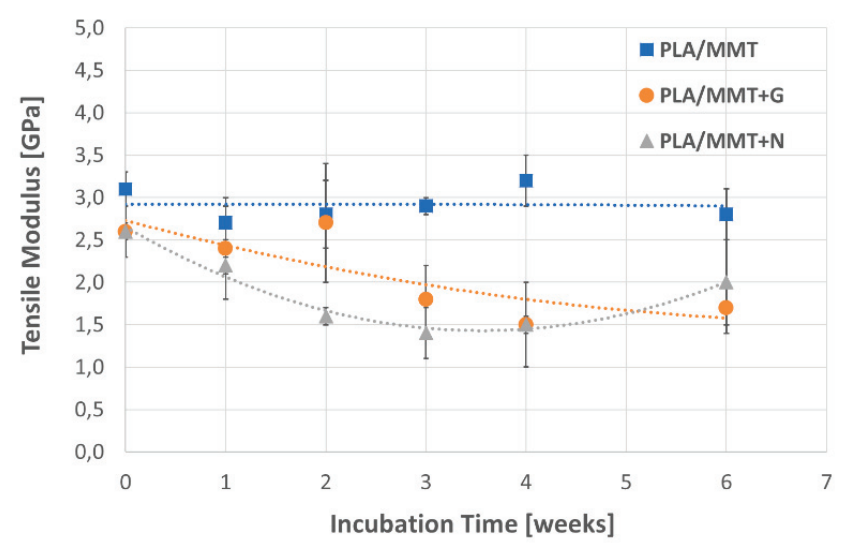

Fig. 3. Changes in elasticity modulus of nanocomposite foils during degradation test

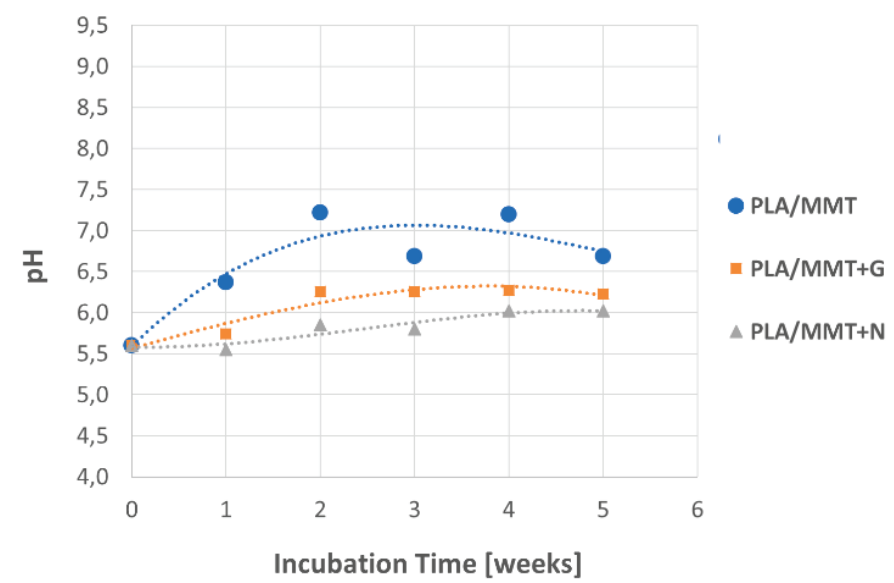

\section{Drug release}

The release of the drugs was monitored by measuring both the $\mathrm{pH}$ and the electrical conductivity of the distilled water in which the samples were immersed, and in Fig. 4 changes in both parameters for the liquid medium after contact with nanocomposite films within 6 weeks of incubation are shown.

It is evident that release increases for two weeks after which it stabilizes, which indicates a high ion exchange capacity of MMT clay. An increase in the $\mathrm{pH}$ value indicates the release of a high amount of ionic substances, and the changes in the ionic conductivity of water are due to the amount of ions. Changes in conductivity in solutions of incubated samples were greatest for $\mathrm{PLA} / \mathrm{MMT}+\mathrm{G}$.

\section{Antibacterial properties \\ before and after degradation process}

The zones inhibiting the growth of $E$. coli in contact with the PLA/MMT + G and PLA/MMT $+\mathrm{N}$ nanocomposite films before a 6-week degradation process are shown in Fig. 5, and the detailed results with the exact diameters of the inhibition zones around the disc samples are summarized in Table 5 (mean values of 6 measurements for each side of the film). As it was already mentioned, the disk samples were placed both smooth and rough sides towards the agar with bacterial culture, so as to obtain information on the effect of the nature of the surface on the amount of antibiotic efficiently released from the film. The smooth and rough surfaces denote the upper and lower sides of the nanocomposite foils, i.e., the "free surface" which does not contact the vessel and the opposite surface in contact with the vessel in which the foils were cast.

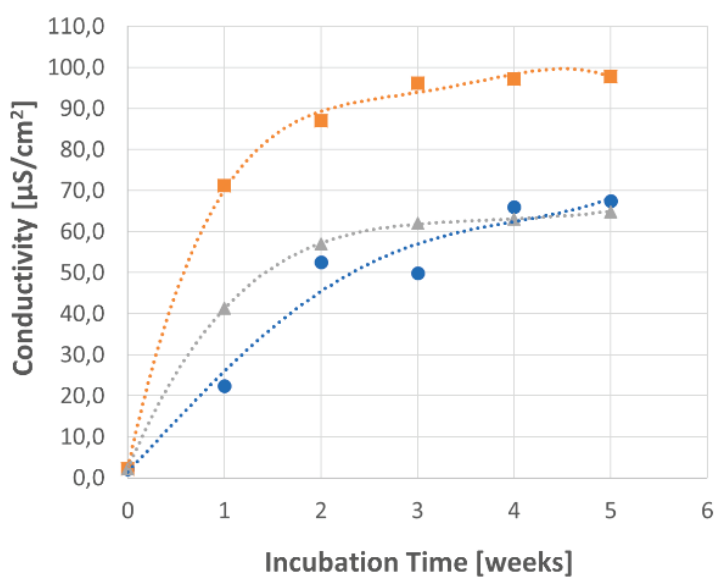

Fig. 4. Changes in $\mathrm{pH}$ and conductivity during a 6-week incubation of nanocomposite films in distilled water at $37^{\circ} \mathrm{C}$ 


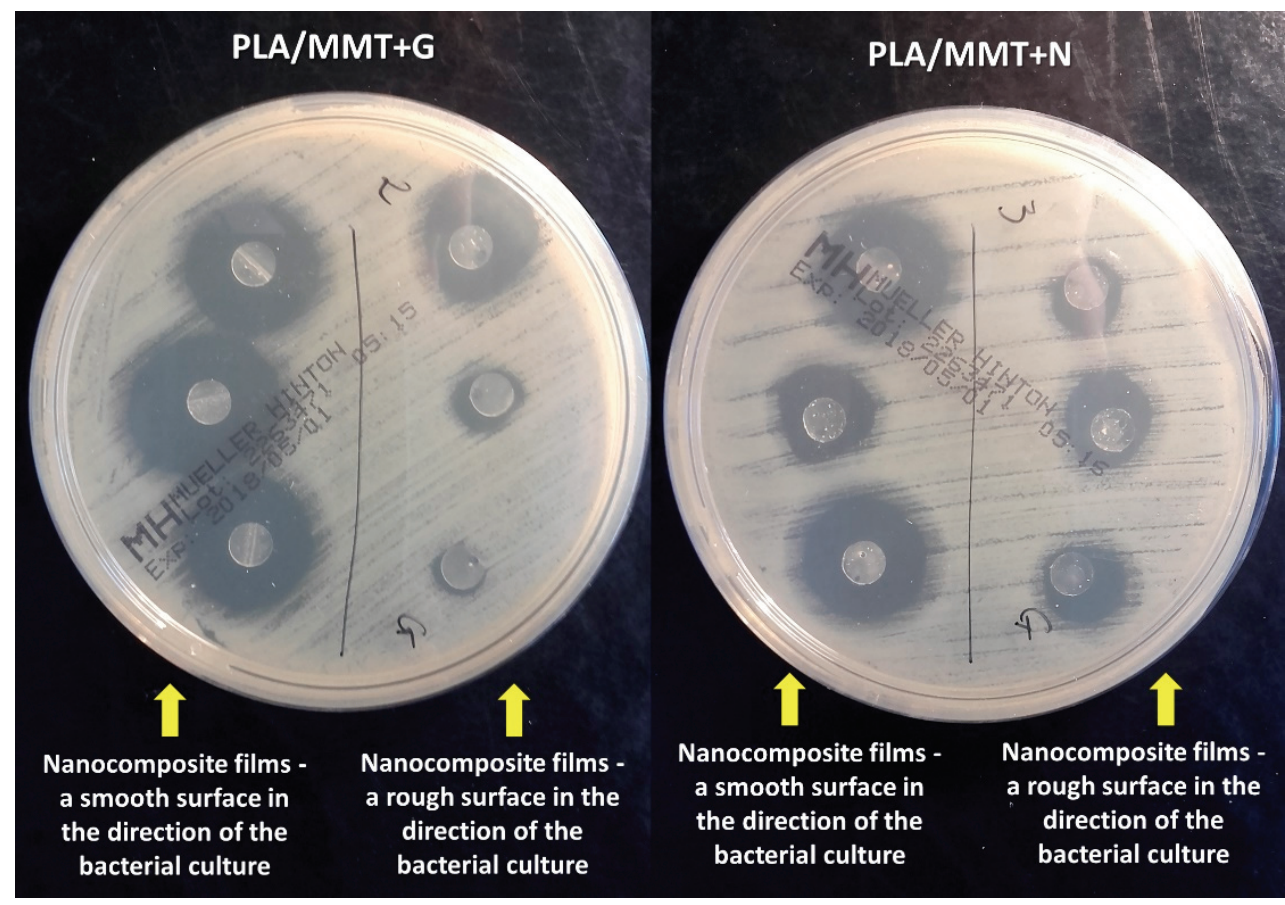

Fig. 5. Antibacterial activity of the PLA/MMT $+\mathrm{G}$ and PLA/MMT $+\mathrm{N}$ nanocomposite films before a 6-week degradation process

Table 5. Zones of $E$. coli growth inhibition around nanocomposite film discs before degradation

\begin{tabular}{|c|c|c|}
\hline \multirow[b]{2}{*}{ Material/Film } & \multicolumn{2}{|c|}{ Zones of inhibition of $E$. coli growth [mm] } \\
\hline & $\begin{array}{l}\text { Rough surface } \\
\text { (upper) }\end{array}$ & $\begin{array}{c}\text { Smooth surface } \\
\text { (lower) }\end{array}$ \\
\hline PLA/MMT & 0 & 0 \\
\hline PLA/MMT + G & 8.0 & 19.0 \\
\hline PLA/MMT + N & 7.6 & 15.3 \\
\hline
\end{tabular}

The antimicrobial activity tests after 6-week degradation were repeated according to the initial goal of this study, although it was difficult to cut disc-shaped samples because nanocomposite films were already partially degraded. Unfortunately, after such a long incubation time it was not possible to distinguish the side of the smooth (starting) foil from the rough side. The samples were thus placed in dishes with bacterial colonies in a random manner, but in this case it was only to capture any symptoms of bactericidal action of nanocomposite materials. Exemplary photos of samples after antibacterial tests are shown in Fig. 6, in which some traces of antibacterial activity are noticeable for both PLA/MMT + G and PLA/MMT + N nanocomposite films after a 6 -week degradation process.

\section{Discussion}

The XRD results indicate that mixing MMT with water does not significantly affect the increase in the interplanar distance in montmorillonite (Table 1), how-

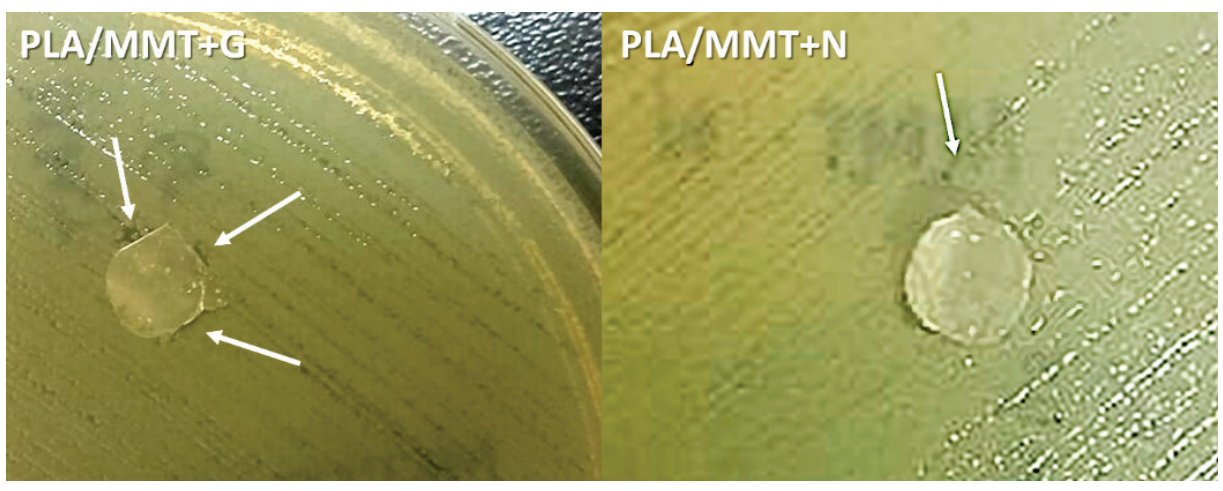

Fig. 6. Antibacterial activity of the PLA/MMT $+\mathrm{G}$ and PLA/MMT $+\mathrm{N}$ nanocomposite films after a 6 weeks degradation in distilled water 
ever, it was confirmed that a single layer of water is located in the interlayer space of MMT, which resulted in an increase in the $d_{001}$ distance by approximately $0.6 \AA$ [17]. In turn, the introduction of an antibiotic, despite it was neomycin or gentamicin, increases the $d_{001}$ values that refer directly to the interlayer distances in these powders (MMT $+\mathrm{G}, \mathrm{MMT}+\mathrm{N})$. The highest $d_{001}$ value was obtained for montmorillonite intercalated with neomycin, for which the intercalation changed the $d_{001}$ value from 11.289 to $16.476 \AA$. The increase in $d_{001}$ values for conjugates with gentamicin and neomycin, by about 4.2 and $5.2 \AA$ respectively, confirmed that both drugs have been successfully intercalated into the interlayer space of MMT. XRD studies of conjugates also indicated that both gentamicin and neomycin sulphates are amorphous substances, which has been confirmed by the lack of diffraction peaks in the entire measurement range.

The powdered montmorillonite, both in neat and hydrated form, revealed a negative electrical potential on the surface of the particles [23]. Intercalation with both gentamicin and neomycin changed the potential of conjugates to a positive one, since both drugs are cationic substances and the change of the potential resulted from their incorporation into the mineral structure. This, in turn, proves the effective MMT intercalation process with these drugs and confirms the results of XRD research. At the same time, the introduction of antibiotics between the interlayer space of montmorillonite lowered the $\mathrm{pH}$ of the powders and while the hydrated montmorillonite was alkaline, the MMT $+\mathrm{G}$ and MMT $+\mathrm{N}$ conjugates were acidic. It is also worth noting that both the Zeta potential and the $\mathrm{pH}$ of gentamicin and neomycin were similar (Table 2).

Nanocomposite films tested for surface hydrophilicity and wettability showed some difference in contact with distilled water. Natural regularity resulting from the preparation method was also found, hence the upper surface of the nanocomposite film was more rough and the contact angle was greater than for the smoother surface on the vessel side (Table 3). Antibiotic additives did not significantly affect the change in wettability, however PLA/MMT + G material had slightly lower contact angle values compared to the reference sample, and the contact angle values for $\mathrm{PLA} / \mathrm{MMT}+\mathrm{N}$ were higher than for the reference material.

The results of the parameters determining the roughness of the PLA/MMT $+\mathrm{G}$ and PLA/MMT $+\mathrm{N}$ nanocomposite films indicate increased values in relation to the reference sample (Table 4). Even if it is taken into account that the results are different de- pending on the tested side of the film, the average deviation of the arithmetic profile suggests that materials filled with $\mathrm{MMT}+\mathrm{G}$ and $\mathrm{MMT}+\mathrm{N}$ conjugates were rougher and the highest roughness was noticed for the PLA/MMT $+\mathrm{G}$ foil. The standard deviation of the $R_{t}$ and $R_{z}$ parameters shows that there are spots with higher or lower roughness in the materials, and the reasons for this phenomenon can be probably found in uneven solvent evaporation at the film preparation stage. Additionally, the increased roughness of foils with MMT conjugates can be explained by the increase in the physical dimensions of a single MMT particle resulting from an increase in the $d_{001}$ distance. As a consequence, randomly oriented MMT particles occupied a larger volume and might have tendency to be more disoriented thus the surface eventually could be more rough.

The mechanical tests, which were carried out throughout the entire degradation experiment, reveal that both the ultimate and long-term properties strongly depended on the type of the filler used. It is visible that the tensile strength at the beginning of the experiment is the highest for the unmodified MMT filler, which may suggest that the neat MMT is capable to improve the mechanical performance in the optimal manner [10], and the not-occupied interlayer space of $11,289 \AA$ is optimal for the compatibility with the PLA matrix and let the polymer chains to be efficiently interlocked by the octahedral layers of MMT. In the foils filled with the intercalated MMT, however, the interlaminar space is most probably no longer freely available, therefore, the mentioned interlocking is not so efficient, thus, the lower mechanical strength is visible even for materials tested at the beginning of degradation experiment. It is, however, worth mentioning that even though the reinforcement by intercalated MMT is not optimal, it is still noticeably effective when the tensile strength values are being compared to the value of the strength of casted foils made of pure PLA, which is about 20-25 MPa [20]. The mentioned strength of the casted PLA foils is pretty low value compared to $60-70 \mathrm{MPa}$ reported by other authors for similar films obtained from PLA [12], but it reflects both the formulation of pure PLA and differences provided by various manufacturing methods The initial trend continues throughout the six-week degradation period and it can be concluded that the efficiency of reinforcement combined with the height of interlaminar space also determine the intensity of volume degradation in nanocomposites. The previously presented hydrolytic degradation mechanism [21] clearly shows that degradation in nanocomposites is dominated by the diffusion of water through 
interfaces which are affected by microstructure of the material with nanofillers, and it may be assumed that the weaker the interfaces are, the more intense diffusion can be expected. In the case of the nanocomposites tested, it was found that the PLA filled with neomycin intercalated MMT has relatively weak interfaces due to the limited ability the polymer chains to be interlocked within the interlayer spaces of the MMT filler. Eventually, it can be concluded that the fastest deterioration of mechanical properties resulting from the degradation process was observed for PLA/MMT $+\mathrm{N}$ foil with the largest interlayer distance and weak interfaces but also with the relatively high surface roughness. The speed of this deterioration is also significant for PLA/MMT $+\mathrm{G}$ foil, with a bit lower interlayer distance but even higher surface roughness. However, after the initial period of degradation, the mechanical behavior of both materials with intercalated fillers appears to stabilize after 4 weeks, suggesting that diffusion still regulates degradation, but the weakening of interfaces reaches a comparable level for both materials, so the driving forces become probably quite similar.

The $\mathrm{pH}$ of distilled water was monitored during a 6-week degradation test (Fig. 4), and reported trends are quite similar to results previously reported by other scientists [2]. It is rather evident that the changes noticed were influenced not by the hydrolytic degradation of PLA, but by the releasing of ions present in the interlayer space of MMT. If hydrolytic degradation of PLA predominated, the $\mathrm{pH}$ of media would decrease since the carboxyl group $(-\mathrm{COOH})$ and hydroxyl group $(-\mathrm{OH})$ occurring as degradation products [4] would have an effect on decreasing the $\mathrm{pH}$ of media. In the case of degradation of PLA/MMT nanocomposites, the release of ions from the inner space of MMT dominates and determines the changes of the $\mathrm{pH}$, and, therefore, the more alkaline the character of MMT is, the more alkaline the surrounding liquid medium becomes (Fig. 4).

The results of antibacterial tests carried out on nanocomposite PLA/MMT films (without drugs) revealed no antibacterial activity of the materials tested. Regardless of the side of the contact of film with the bacterial culture, a zero zone of inhibition of bacterial growth around the samples was observed on both the rough and the smooth side of the foils.

It was worth mentioning that no intrinsic antibacterial properties have been reported for neat PLA/MMT foils (with no drug intercalation), even if they were previously reported for this type of material [20]. This can easily be explained by the lack of ultrasonic mixing, which determined the complete exfoliation occur- ring in MMT clay, leading to the antibacterial effect of the resulting nanocomposite.

However, interesting results were observed for nanocomposite films in which MMT was intercalated with gentamicin or neomycin (Fig. 5). Both types of materials showed antibacterial activity manifested by the occurrence of zones inhibiting the growth of Gramnegative bacteria. In this case, there is also a clear tendency for large zones of inhibition to appear around the film discs, which were placed with the smooth side towards the bacterial culture. The rough surface gave an average of half the diameters of the inhibition zones, as well as - what is characteristic - irregular zones of an elliptical shape. Therefore, it can be assumed that the appearance of larger zones of inhibition around the foil samples with the smooth surfaces was probably the result of a better adhesion of the smooth side of the film to the agar with the bacterial culture.

In addition, it is worth noting that the presence of $E$. coli growth inhibition zones around the nanocomposite films PLA/MMT $+\mathrm{G}$ and PLA/MMT $+\mathrm{N}$ unambiguously indicates that the interaction of both antibiotics with the clay carrier during intercalation and also with the polymer matrix during the manufacturing of nanocomposites did not adversely affect their bactericidal effect.

What is equally important from a utilitarian point of view, PLA/MMT $+\mathrm{G}$ and PLA/MMT $+\mathrm{N}$ films were re-tested for antimicrobial activity after 6 weeks of degradation in distilled water at $37{ }^{\circ} \mathrm{C}$ to check whether there is still a biocidal effect from antibiotics or whether the entire antibiotic was released into the incubating medium during this period. Figure 6 shows that even after such a long incubation period, there is still a biocidal effect and for both types of nanocomposite films PLA/MMT $+\mathrm{G}$ and PLA/MMT $+\mathrm{N}$ a small area was recorded without bacterial growth in the immediate vicinity of the discs. This effect indicates a residual zone of inhibition, resulting from the presence of small amounts of antibiotic diffusing into the agar, however, these results indicate that after such a long incubation/degradation period, antibacterial effects of this type of nanocomposite materials can be expected.

\section{Conclusions}

It may be finally concluded that the PLA/MMT nanocomposite films tested can be successfully intercalated both with gentamicin and neomycin, obtaining very good antibacterial properties, thus their use in 
biomedical applications can be significantly extended. In addition, drug-intercalated nanocomposite films tend to degrade faster due to weakened interfaces and limited interlocking between clay particles and the polylactide matrix. This may have a significant practical effect, especially in applications where controlled degradation time is crucial.

\section{Acknowledgements}

This study was performed within the framework of funding for statutory activities of AGH University of Science and Technology in Cracow, Faculty of Materials Science and Ceramics (11.11.160.617).

\section{References}

[1] AzHar F.F., OlaD A., A study on sustained release formulations for oral delivery of 5-fluorouracil based on alginatechitosan/montmorillonite nanocomposite systems, Appl. Clay Sci., 2014, 101, 288-296.

[2] BARTKOWIAK-JowsA M., BĘDZiŃSKI R., SzARANieC B., CHŁOPEK J., Mechanical, biological, and microstructural properties of biodegradable models of polymeric stents made of PLLA and alginate fibers, Acta Bioeng. Biomech., 2011, 13, 21-28.

[3] Cypes S.H., Saltzman W.M., Giannelis E.P., Organosilicate-polymer drug delivery systems: controlled release and enhanced mechanical properties, J. Contr. Release, 2003, 90, 163-169.

[4] Dong W., Zou B., Yan Y., Ma P., Chen M., Effect of chainextenders on the properties and hydrolytic degradation behavior of the poly(lactide)/poly(butylene adipate-co-terephthalate) blends, Int. J. Molec. Sci., 2013, 14, 20189-20203.

[5] Dziadkowiec J., Mansa R., Quintela A., Rocha F., Detellier C., Preparation, characterization and application in controlled release of ibuprofen-loaded guar gum/montmorillonite bionanocomposites, Appl. Clay Sci., 2017, 135, 52-63.

[6] Fejer I., Kata M., Eros I., Dekany I., Interaction of monovalent cationic drugs with montmorillonite, Colloid Polym. Sci., 2002, 280, 372-379.

[7] Feng S.S., Mei L., Anitha P., Gan C.W., Zhou W., Poly(lactide)-vitamin E derivative/montmorillonite nanoparticle formulations for the oral delivery of Docetaxel, Biomater., 2009, 30, 3297-3306.

[8] Golubeva O.Y., Pavlova S.V., Adsorption of thiamine hydrochloride (Vitamin B1) by synthetic layered silicates with a montmorillonite structure, Glass Phys. Chemist., 2014, 40, $375-379$.

[9] Jafarbeglou M., Abdouss M., Shoushtari A.M., JAFARBEGLOU M., Clay nanocomposites as engineering drug delivery systems, RSC Adv., 2016, 55, 50002-50016.
[10] Jo B.W., PARK S.K., Kim D.K., Mechanical properties of nano-MMT- reinforced polymer composite and polymer concrete, Constr. Build. Mater., 2008, 22 (1), 14-20.

[11] Kant A., DAtTA M., Extended release of metronidazole using montmorillonite as drug delivery vehicle, J. Indian Chem. Soc., 2016, 93, 805-807.

[12] Kobielarz M., Gazińska M., Tomanik M., StęPak B., Szustakiewicz K., FilipiaK J., AntończaK A., Pezowicz C., Physicochemical and mechanical properties of $\mathrm{CO}_{2}$ laser-modified biodegradable polymers for medical applications, Polym. Degrad. Stabil., 2019, 165, 182-195.

[13] MClaren A.D., Peterson G.H., Montmorillonite as a caliper for the size protein molecules, Nature, 1961, 192, 960-961.

[14] Meng N., Zhou N., Zhang S., Shen J., Synthesis and antimicrobial activities of polymer/montmorillonite-chlorhexidine acetate nanocomposite films, Appl. Clay Sci., 2009, 42, 667-670.

[15] Miotke M., Strankowska J., Kwela J., Strankowski M., PiszCZYK L., JóZEFowicz M., GAZDA M., Nanosize effect of clay mineral nanoparticles on the drug diffusion processes in polyurethane nanocomposite hydrogels, Eur. Phys. J. Plus, 2017, 132, 401-416.

[16] Nunes C.D., VAZ P.D., Fernandes A.C., Ferreira P., Loading and delivery of sertraline using inorganic micro- and mesoporous materials, Eur. J. Pharm. Biopharm., 2007, 66, 357-365.

[17] Ponguanyakul T., Priprem A., Puttipipatkhachorn S., Investigation of novel alginate-magnesium aluminum silicate microcomposite films for modified-release tablets, J. Contr. Release, 2005, 107, 343-356.

[18] Rapacz-Kmita A., Stodolak-ZYch E., BućKo M.M., MikolajCZYK M., Dudek P., TRYBus M., Characterisation, in vitro release study, and antibacterial activity of montmorillonite-gentamicin complex material, Mater. Sci. Eng. C, 2017, 70, 471-478.

[19] RapaCZ-KMita A., GajeK M., DudeK M., StodolaK-Zych E., SZARANIEC B., LACH R., Thermal, structural and mechanical analysis of polymer/clay nanocomposites with controlled degradation, J. Therm. Anal. Calorim., 2017, 127, 389-398.

[20] Rapacz-Kmita A., Pierchala M.K., Tomas-Trybuś A., SZARANIEC B., KARWOT J., The wettability, mechanical and antimicrobial properties of polylactide/montmorillonite nanocomposite films, Acta Bioeng. Biomech., 2017, 19, 25-33.

[21] RapacZ-Kmita A., StodolaK-Zych E., SzaranieC B., GAJEK M., DUDEK P., Effect of clay mineral on the accelerated hydrolytic degradation of polylactide in the polymer/clay nanocomposites, Mater. Lett., 2015, 146, 73-76.

[22] Salahuddin N., Kenawy E.-R., Abdeen R., Polyoxypropylene-montmorillonite nanocomposites for drug delivery vehicles: Preparation and characterization, J. Appl. Polym. Sci., 2012, 125, 157-166.

[23] UdDIN F., Montmorillonite: An introduction to properties and utilization, [in:] Current Topics in the Utilization of Clay in Industrial and Medical Applications, M. Zoveidavianpoor (Ed.), Intech Open, London, 2018, 4-23.

[24] Yuan Q., Shah J., Hein S., Misra R., Controlled and extended drug release behavior of chitosan-based nanoparticle carrier, Acta Biomater., 2010, 6, 1140-1148. 